\title{
OS FOSFATOS ALUMINOSOS DA SÉRIE DA CRANDALLITA - UMA REVISÃO
}

\author{
Maria Cristina Motta de TOLEDO
}

\begin{abstract}
RESUMO
Os minerais da série da crandallita têm fórmula geral $\mathrm{A} \mathrm{Al}_{3}\left(\mathrm{PO}_{4}\right)\left(\mathrm{PO}_{3} \mathrm{OH}\right)(\mathrm{OH})_{6}$, onde A são cátions grandes como $\mathrm{Na}^{+}, \mathrm{K}^{+}, \mathrm{Ca}^{2+}, \mathrm{Pb}^{2+}, \mathrm{Ba}^{2+}, \mathrm{Sr}^{2+}, \mathrm{ETR}^{3+}$, e outros, em coordenação 12, podendo apresentar diversas substituições catiônicas e aniônicas. Formam-se comumente durante o intemperismo sobre diversos materiais originais com disponibilidade em P. De grande estabilidade no ambiente superficial, podem ocorrer também em depósitos detríticos. Estes minerais têm sido mencionados como uma das principais fontes de $\mathrm{P}$ em solos e o reconhecimento de sua ampla distribuição também em ambientes sedimentares marinhos tem crescido, modificando as idéias mais antigas sobre as quantidades de $\mathrm{P}$ nesta etapa de seu ciclo. Os fosfatos crandallíticos são geralmente considerados prejudiciais ao aproveitamento dos minérios apatíticos aos quais se associam. No Brasil não são considerados como fonte de fósforo para fertilizantes, embora haja registro desta aplicação em outros países. Por outro lado, sua estrutura aberta bem como a alta estabilidade e relativa abundância nas formações superficiais podem representar argumentos suficientes para a busca de aplicações em diferentes setores. Esta revisão apresenta uma síntese da bibliografia a respeito dos aspectos mineralógicos, geoquímicos, cristaloquímicos e genéticos dos minerais desta série.
\end{abstract}

Palavras-chave: Crandallita, intemperismo, mineralogia, fosfatos.

\begin{abstract}
Crandallite series minerals have general formula $\mathrm{A} \mathrm{Al}_{3}\left(\mathrm{PO}_{4}\right)\left(\mathrm{PO}_{3} \mathrm{OH}\right)(\mathrm{OH})_{6}$, where $\mathrm{A}$ are big cations like $\mathrm{Na}^{+}, \mathrm{K}^{+}, \mathrm{Ca}^{2+}, \mathrm{Pb}^{2+}, \mathrm{Ba}^{2+}, \mathrm{Sr}^{2+}, \mathrm{ETR}^{3+}$, and others with coordination 12 , and several cationic and anionic substitutions may occur. They are often formed by weathering processes on different P-rich original materials. Detritical deposits may also contain crandallite minerals, because of their high stability. They have been mentioned as the main source of phosphorus in soils. The recent increase in their recognition in marine sediments may modify former ideas about $\mathrm{P}$ quantities in this stage of its geochemical cycle. Crandallite phosphates, when associated with phosphatic ore, are generally considered harmful to mining and processing. In Brazil, unlike other countries, they are not considered as raw material for phosphatic fertilizers. Their open structure, high stability, and relative abundance may represent enough arguments to find applications in different sectors. This paper presents a bibliographic review of mineralogical, geochemical and genetic aspects of the minerals of this series.
\end{abstract}

Keywords: Crandallite, weathering, mineralogy, phosphates.

\section{INTRODUÇÃO}

Esta revisão reúne informações dispersas na literatura a respeito da variabilidade de composição e outros dados importantes como estrutura, comportamento térmico e modo de ocorrência dos minerais mais comuns da série da crandallita, minerais estes que possuem uma distribuição importante nas formações superficiais sobre vários tipos de rochas e que, apesar de não terem sido até o momento utilizados de forma extensiva como recurso mineral, apresentam algumas potencialidades de aplicação, bem como desempenham papel destacado na dinâmica de elementos presentes em seus meios de formação.

Os fosfatos aluminosos da série da crandallita ocorrem comumente em perfis de intemperismo sobre materiais originais com disponibilidade em 
$\mathrm{P}$, principalmente rochas sedimentares tipo fosforitos marinhos e também outros depósitos sob influência de guano, sobre rochas ígneas, sobretudo as alcalinas, carbonatíticas e pegmatíticas, e rochas metamórficas. Também ocorrem como resultado de alteração hidrotermal e ainda como minerais neogenéticos em sedimentos marinhos.

Estes minerais têm sido mencionados como uma das principais fontes de $\mathrm{P}$ em solos (NORRISH 1968) e o reconhecimento de sua ampla distribuição tem crescido. RASMUSSEN (1996) adverte para o fato de que, dada sua ampla ocorrência em sedimentos arenosos marinhos recentes na Austrália, devem constituir um reservatório importante de $\mathrm{P}$ no ambiente marinho, não reconhecido antes provavelmente devido a suas baixas concentrações e às pequenas dimensões de seus cristais. Suas estimativas indicam que o $\mathrm{P}$ removido do oceano pela precipitação destes minerais pode ser tão importante como outros reservatórios como a carbonatofluorapatita e o fósforo orgânico.

Particularmente nos mantos de alteração laterítica sobre complexos carbonatíticos, ricos em fosfatos primários e com disponibilidade nos cátions potencialmente presentes em sua fórmula estrutural, aqueles fosfatos supérgenos são abundantes e distribuem-se nos perfis evidenciando as características geoquímicas das rochas originais e a mobilidade dos elementos envolvidos (por exemplo MCKIE 1962, na África e LOTTERMOSER 1990, na Austrália, entre outros).

No Brasil, têm sido descritos como resultado de neoformações durante os ciclos de desestabilização e reprecipitação de fosfatos em perfis lateríticos sobre rochas ígneas em complexos alcalino-carbonatíticos (SOUBIÈS et al. 1991, MELFI et al. 1991, LEMOS 1994, PEREIRA 1995, CORREAA 1996, ALCOVER NETO \& TOLEDO 1993 e TOLEDO et al. 1997, 1999), pegmatitos (SVISERO et al. 1984, CORREIANEVES et al. 1987, CASSEDANE \& NEN 1991), rochas metamórficas (COSTA 1984, OLIVEIRA \& COSTA 1984, VISSER et al. 1997) e materiais sedimentares como fosforitos ou outros materiais de origem orgânica (COSTA 1984, OLIVEIRA \& COSTA 1984). Ocorrem também como mineral detrítico em aluviões no estado de Minas Gerais, como citado por DEVISMES et al. (1968), comparando com ocorrências semelhantes no Gabão e na França e também por PALACHE et al. (1951). Particularmente para os fosfatos da série da crandallita existentes em Tapira, SOUBIÈS et al. (1991) destacou seu importante papel como fase portadora dos ETR liberados no perfil pela alteração da apatita primária.

No Brasil, os fosfatos da série da crandallita não são considerados como fonte de fósforo para fertilizantes, embora haja registro desta aplicação em outros países (HILL et al. 1950, DOAK et al. 1965, LEHR 1967, GILKES \& PALMER 1979, HOARE 1980). Estes minerais são geralmente considerados prejudiciais ao aproveitamento dos minérios apatíticos aos quais freqüentemente se associam, dificultando a concentração do mineral de minério, abaixando sua qualidade. Por outro lado, sua estrutura aberta, com grandes cavidades entre as folhas de octaedros centrados em átomos de $\mathrm{Al}$, bem como a alta estabilidade e relativa abundância nas formações superficiais podem vir a representar argumentos suficientes para a busca de aplicações em diferentes setores.

\section{COMPOSIÇÃO, CLASSIFICAÇÃO, ESTRUTURA}

Os minerais da série da crandallita, de fórmula geral simplificada $\mathrm{A} \mathrm{Al}_{3}\left(\mathrm{PO}_{4}\right)$ $\left(\mathrm{PO}_{3} \mathrm{OH}\right)(\mathrm{OH})_{6}$, também mencionados como grupo ou família da plumbogummita, recebem tradicionalmente denominações particulares em função do cátion predominante na posição $\mathrm{A}$ (Ca: crandallita, Sr: goyazita, Pb: plumbogummita, Ba: gorceixita, ETR: florencita), podendo formar solução sólida entre vários de seus membros.

A crandallita tem uma estrutura hexagonal consistindo de octaedros de $\mathrm{Al}(\mathrm{OH})_{4} \mathrm{O}_{2}$ ligados em anéis hexagonais ou trigonais, formando folhas perpendiculares ao eixo $\mathrm{c}$. Cada tetraedro $\mathrm{PO}_{4}$ compartilha três vértices com três octaedros de um anel; os vértices não compartilhados formam pontes de hidrogênio com os vértices dos tetraedros das folhas adjacentes; os cátions da posição $\mathrm{A}$, rodeados assimetricamente por 12 ânions oxigênio e hidroxila, acomodam-se em grandes cavidades entre as folhas (BLOUNT 1974) (figura 1). 

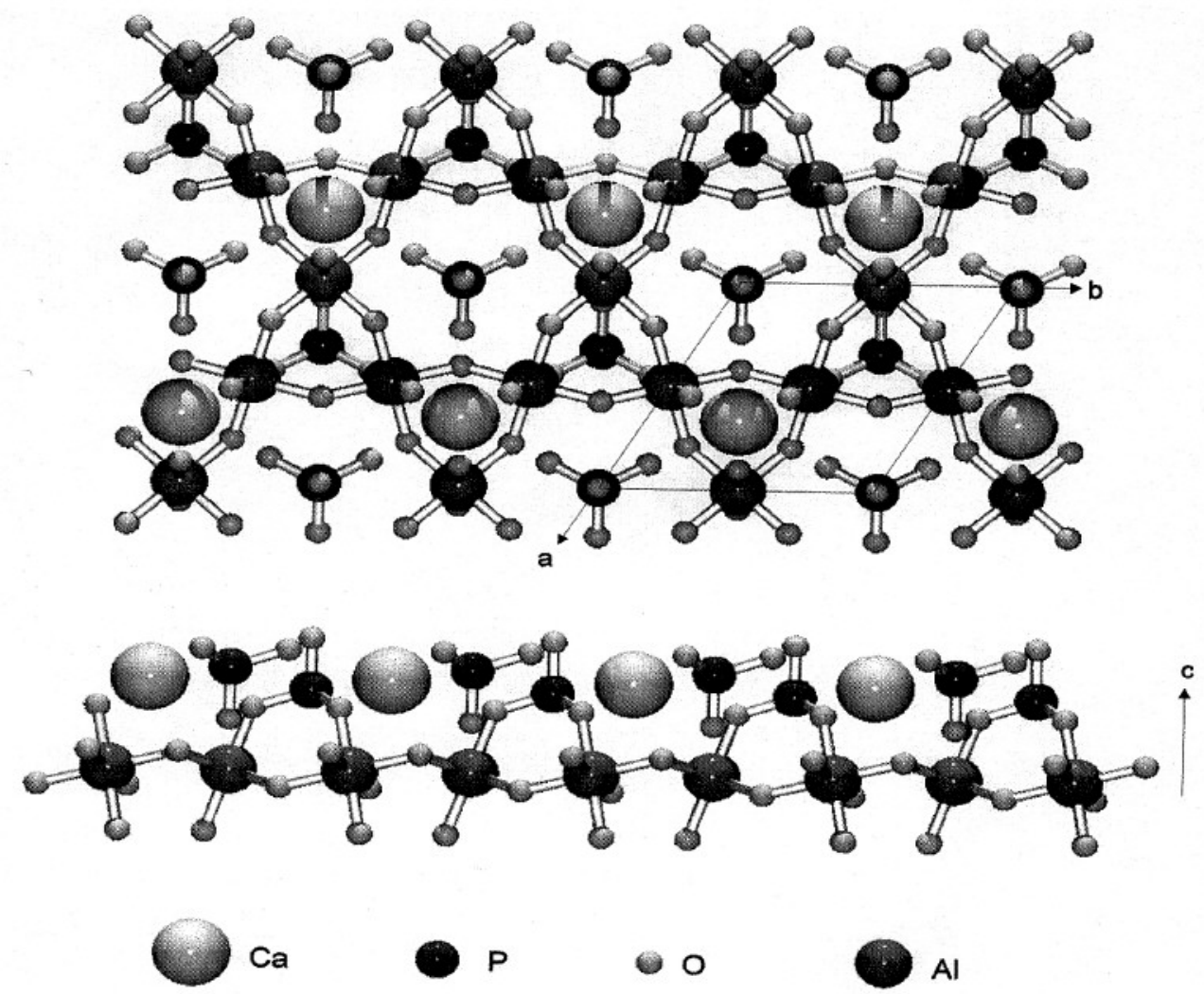

Al

FIGURA 1 - Esquema da estrutura da crandallita segundo BLOUNT (1974), elaborado com o auxílio do programa ORTEP3 (FARRUGIA 1997), por FERRARI (2000).

HENDRICKS (1937), seguido por WANG et al. (1965), BLOUNT (1974) e KATO (1987, 1990) determinaram a estrutura dos minerais desta grande família, reconhecendo o grupo espacial $R-3 m$. Segundo KATO (1971), tanto o grupo espacial descentrado $R 3 \mathrm{~m}$ como o centrado $R-3 m$ são consistentes com os dados difratométricos da goyazita; o refinamento da estrutura feito por este autor foi bem sucedido considerando o grupo $R-3 m$, concluindo ele que este seria o grupo espacial mais provável para esta estrutura. RADOSLOVITCH \& SLADE (1980) indicam o grupo $R 3 m$ e RADOSLOVICH (1982) considera $\mathrm{Cm}$ o grupo espacial dos membros com cátions trivalentes na posição A. BLACKBURN \& DENNEN (1997) consideram os seguintes sistemas cristalinos e grupos espaciais para os minerais da série: crandallita (trigonal, $R 3 \mathrm{~m}$ ), gorceixita (monoclínico, $\mathrm{Cm}$ ), goyazita (trigonal, $R-3 m$.), florencita (trigonal, $R 3 m$ ) $\mathrm{e}$ plumbogummita (trigonal, $R 3 m$ ). As fichas ICDD mais antigas da gorceixita -- 19-535 e 33-130, que a consideravam respectivamente romboédrica grupo espacial $R-3 m$ e monoclínica
$\mathrm{Cm}$-- foram substituídas pela 41-1459, que a considera também monoclínica $\mathrm{Cm}$.

São isoestruturais com a alunita, formando uma grande família de sulfatos, arsenatos e fosfatos de fórmula geral $\mathrm{A} \mathrm{B}_{3}\left(\mathrm{XO}_{4}\right)_{2}(\mathrm{OH}, \mathrm{F})_{6}$, ou $\mathrm{A} \mathrm{B}_{3}\left(\mathrm{XO}_{4}\right)_{2}(\mathrm{OH}, \mathrm{F})_{5} \mathrm{H}_{2} \mathrm{O}$ ou ainda $\mathrm{A} \mathrm{B}_{3}$ $\left(\mathrm{XO}_{4}\right)\left(\mathrm{XO}_{3} \mathrm{OH}\right)(\mathrm{OH}, \mathrm{F})_{6}$, cuja classificação ainda está por ser completamente sistematizada, onde:

A: cátions grandes como $\mathrm{Na}^{+}, \mathrm{K}^{+}, \mathrm{Ag}^{+}$, $\mathrm{NH}^{4+}, \mathrm{H}_{3} \mathrm{O}^{+}, \mathrm{Ca}^{2+}, \mathrm{Pb}^{2+}, \mathrm{Ba}^{2+}, \mathrm{Sr}^{2+}, \mathrm{Ce}^{3+}, \mathrm{ETR}^{3+}$, em coordenação 12

B: cátions como $\mathrm{Al}^{3+}, \mathrm{Fe}^{3+}, \mathrm{Cu}^{2+} e \mathrm{Zn}^{2+}, \mathrm{em}$ coordenação tetraédrica

$\mathrm{XO}_{4}: \mathrm{SO}_{4}{ }^{2-}, \mathrm{PO}_{4}{ }^{3-}, \mathrm{ASO}_{4}{ }^{3-}, \mathrm{CO}_{3}{ }^{2-}, \mathrm{SbO}_{4}{ }^{3-}$, $\mathrm{CrO}_{4}^{2-} \mathrm{Ou} \mathrm{SiO}_{4}^{4-}$

Outros elementos já foram encontrados no sítio A, como U e Th (VAN WAMBEKE 1972), U (MICHEL et al. 1982) e W (NICOLAS \& DE ROSEN 1963). Podem ocorrer vacâncias estruturais; MCKIE (1962) e GRIEFFO et al. (1984) apontam deficiências de, respectivamente, 31 e $10 \%$ no sítio A; LOTTERMOSER (1990) encontrou deficiências no sítio $\mathrm{XO}_{4}$, excessos 
no sítio B e comportamento irregular (deficiências e excessos) no sítio A. Apenas TAYLOR et al. (1984) encontraram fórmulas estruturais próximas da fórmula da crandallita anidra $\mathrm{A} \mathrm{B}_{3}$ $\left(\mathrm{PO}_{4}\right)_{2} \mathrm{O}_{2,5}$ sem vacâncias ou excessos.

A série crandallita pode ser incluída na família alunita-jarosita por semelhança estrutural. Há freqüentes registros de soluções sólidas no sítio A nesta família, sendo que substituições nos sítios $\mathrm{B}$ e $\left(\mathrm{XO}_{4}\right)$ são menos documentadas. $\mathrm{O}$ grupo aniônico $\mathrm{XO}_{4}$ tem sido usado para definir três classes, com a forte relação entre a ocupação $\mathrm{A}$ e o ânion $\mathrm{XO}_{4}$ refletindo mecanismos de compensação de cargas, que incluem a protonação de um dos ânions trivalentes, se necessário, como no caso da gorceixita (PALACHE et al. 1951):

- grupo da alunita, onde o ânion $\mathrm{XO}_{4}$ é divalente e os cátions são geralmente monovalentes,

- grupo da beudantita ou woodhouseíta, onde há um ânion $\mathrm{XO}_{4}$ divalente e outro trivalente, e cátions geralmente divalentes e

- grupo da plumbogummita, crandallita ou goyazita, onde os dois ânions $\mathrm{XO}_{4}$ são trivalentes e os cátions são di ou trivalentes.

O mesmo autor já citado, SCOTT (1987), estudando minerais da família alunita-jarosita em gossans relacionados à mineralização em $\mathrm{Pb}$ Zn do Monte Isa, na Austrália, notou que ocorre uma extensiva solução sólida entre fases com diferentes composições nos sítios $\mathrm{A}, \mathrm{B}$ e $\left(\mathrm{XO}_{4}\right)$, com freqüente correlação entre os íons das posições $\mathrm{A}$ e $\mathrm{XO}_{4}$. A partir de seus dados, elaborou uma organização na classificação dos minerais da família alunita-jarosita, mantendo a nomenclatura de PALACHE et al. (1951), com ligeiras modificações. Propõe uma primeira divisão em dois supergrupos com base na ocupação predominante do sítio $\mathrm{B}$ (supergrupo da alunita se $\mathrm{Al}>\mathrm{Fe}$ e supergrupo da jarosita se $\mathrm{Fe}>\mathrm{Al}$ ) e, a partir daí, uma divisão em grupos segundo a ocupação do sítio A por cátions mono, di ou trivalentes, paralelamente à ocupação do sítio $\left(\mathrm{XO}_{4}\right)$. Em suas considerações sobre esta divisão, cita a ausência, até aquela data, de equivalentes do grupo da florencita $\left(\mathrm{A}=\mathrm{ETR}, \mathrm{B}=\mathrm{Al}\right.$ e $\left.\mathrm{XO}_{4}=\mathrm{PO}_{4}\right)$ com $\mathrm{Fe}$ na posição $\mathrm{B}$.

No que se refere à solução sólida no sítio A dos membros fosfáticos, há substituições documentadas envolvendo cátions de diferentes valências entre os membros extremos: florencita $\mathrm{CeAl}_{3}\left(\mathrm{PO}_{4}\right)_{2}(\mathrm{OH})_{6}$, crandallita $\mathrm{CaAl}_{3} \mathrm{H}\left(\mathrm{PO}_{4}\right)_{2}(\mathrm{OH})_{6}$, gorceixita $\mathrm{BaAl}_{3} \mathrm{H}\left(\mathrm{PO}_{4}\right)_{2}(\mathrm{OH})_{6}$, goyazita $\mathrm{SrAl}_{3} \mathrm{H}\left(\mathrm{PO}_{4}\right)_{2}(\mathrm{OH})_{6} \mathrm{e}$ plumbogummita $\mathrm{PbAl}_{3} \mathrm{H}\left(\mathrm{PO}_{4}\right)_{2}(\mathrm{OH})_{6}$.

A substituição do Ce trivalente por íons divalentes pode ser balanceada seja pela substituição de $\mathrm{PO}_{4}{ }^{3-}$ por $\mathrm{SO}_{4}{ }^{2-}$ ou pela protonação do ânion fosfato. Este mecanismo modifica o comprimento das ligações $\mathrm{P}$ - $\mathrm{O}$ a ponto de modificar o grupo espacial, transformando uma estrutura $R-3 m$ em $C m$ (RADOSLOVICH 1982). Por isso, na classificação adotada por SCOTT (1987), o grupo da florencita se distingüe do grupo da plumbogummita.

JAMBOR (1999) sugeriu, para a nomenclatura dos minerais do grupo da beudantita, a utilização de triângulos (As - P - S) para cada cátion dominante na posição $\mathrm{A}(\mathrm{Pb}, \mathrm{Ba}, \mathrm{Sr}$ ou $\mathrm{Ca})$, considerando a proporção $\mathrm{Al} / \mathrm{Fe}$ na posição $\mathrm{B}$ (um triângulo para $100 \%$ de $\mathrm{Al}$ e outro paralelo para $100 \%$ de Fe, superpostos, de modo a possibilitar a visualização da proporção $\mathrm{Fe} / \mathrm{Al}$ na ocupação da posição $\mathrm{B}$, representada pela distância do ponto considerado em relação aos dois triângulos).

As estruturas da alunita e da crandallita $(R-3 m)$ foram consideradas por GOREAUD \& RAVEAU (1980) similares à estrutura dos pirocloros $(F d 3 m)$, que pode ser descrita de forma equivalente a uma cela romboédrica com as mesmas dimensões da alunita e caracterizada pelo mesmo grupo espacial $R-3 m$.

MCKIE (1962), estudando minerais do grupo da plumbogummita de ocorrência natural, encontrou uma relação direta entre os parâmetros $a$ e $c$ e a média do raio iônico dos cátions da posição A. Este autor determinou os parâmetros $a$ e $c$ através das reflexões (020) e (033):

$d_{(020)}=a / 4 \quad$ e $\quad d_{(033)}=a \cdot c\left(9 \cdot a^{2}+12 \cdot c^{2}\right)^{-1 / 2}$

MENCHETTI \& SABELLI (1976), estudando alunita, jarosita, natroalunita e natrojarosita sintéticas, também mostraram que as variações nos parâmetros de cela unitária dependem das substituições isomórficas; a substituição do cátion trivalente central dos octaedros $\left(\mathrm{Al}^{3+}\right.$ por $\mathrm{Fe}^{3+}$ na posição B) modifica o valor de $a$, enquanto $c$ permanece constante; ao contrário, a substituição do cátion da posição A causa modificação de $c$, sem modificar $a$, o que não está totalmente em acordo com MCKIE (1962).

GILKES \& PALMER (1983) ressaltaram que, não tendo MCKIE (1962) incluído em seu 
estudo membros ricos em $\mathrm{Ca}$, a relação encontrada predizia valores muito menores que os reais para $a$ e $c$ de crandallita. Em estudo de crandallita e goyazita sintéticas, aqueles autores calcularam os parâmetros de cela unitária a partir apenas das reflexões 303 e 220 , devido à incerteza quanto à indexação de várias outras reflexões, usando o mesmo procedimento adotado por MCKIE (1962). Concluíram que a dimensão $c$ aumenta linearmente com o aumento da proporção molar de Sr na molécula e que a dimensão $a$ aumenta fracamente. Os valores por eles encontrados encontram-se na tabela 1 , juntamente com valores dos parâmetros $a$ e $c$ para vários minerais da série da crandallita e alguns compostos sintéticos similares encontrados na literatura. As discrepâncias encontradas entre valores obtidos para minerais naturais e seus correspondentes sintéticos podem encontrar explicação na presença de substituições isomórficas nos espécimes naturais (GILKES \& PALMER 1983).

$\mathrm{Na}$ mesma pesquisa, combinando os estudos de ATD com os cálculos dos parâmetros de cela unitária, estes últimos autores concluíram que a desidratação da crandallita é acompanhada por contração da dimensão $c$ e por expansão na dimensão $a$. Afirmam ainda que a maior sensibilidade do parâmetro $c$ com a entrada de $\mathrm{Sr}$ na estrutura e também com a desidroxilação ou desidratação deve ser devida à rigidez das folhas contínuas dos octaedros $\mathrm{Al}(\mathrm{OH})_{4} \mathrm{O}_{2}$, paralelas ao plano (001); por outro lado, a ligação destas folhas na direção $c$, sendo feita através de pontes de hidrogênio fracas entre os ânions apicais $(\mathrm{OH})_{1 / 2} \mathrm{O}_{1 / 2}$ de tetraedros $\mathrm{PO}_{31 / 2}(\mathrm{OH})_{1 / 2}$ não ligados (não formam filas, nem folhas, nem arcabouços tridimensionais), é mais fraca, permitindo mais facilmente alguma flexibilidade.

SCOTT (1987) também mencionou o problema da variação dos parâmetros, afirmando que a ocupação do sítio $\mathrm{B}$ pelo $\mathrm{Fe}^{3+}$ ou pelo $\mathrm{Al}^{3+}$ influencia o parâmetro $a$, não quantificando, no entanto, esta variação.

A estabilidade da estrutura da crandallita é favorecida, segundo SCHWAB et al. (1990a, 1990 b), por cátions maiores que o Ca, por adaptarem-se melhor na coordenação 12 nos grandes poliedros $6(\mathrm{OH}) 60$ que rodeiam os cátions. Assim, $\mathrm{Sr}, \mathrm{Pb}$ e $\mathrm{Ba}$, com seus raios iônicos respectivamente de $1,44 \AA, 1,49 \AA$ e $1,61 \AA$ são bem recebidos na estrutura que apresenta parâmetro $c$ de $17,275 \AA$ para gorceixita a $16,611 \AA$ para goyazita, em correlação linear com os raios iônicos. Extrapolando-se a curva de correlação, seria obtido um valor de $16,20 \AA$ para a crandallita puramente cálcica; embora crandallita natural apresente valores de $c$ próximos a este, não é possível sintetizar esta fase totalmente livre de substituintes do cálcio. O raio iônico do Ca seria muito pequeno e seu potencial iônico fraco demais para contrair a estrutura para valores de $c$ de 16,20 $\AA$ formando uma cavidade adequada para sua dimensão. Crandallita pura não seria, então, estável. Entretanto, se o Ca for substituído por um íon trivalente de dimensão semelhante, como o $\mathrm{Ce}^{3+}(1,34 \AA)$, então o alto potencial iônico seria suficiente para contrair e estabilizar a estrutura.

\section{CARACTERÍSTICAS MORFOLÓGICAS E ÓPTICAS}

Os fosfatos crandalíticos descritos na literatura apresentam-se com morfologia variada, tendo sido citada a existência de cristais romboédricos, aciculares, tetraédricos ou hexagonais, geralmente submilimétricos a criptocristalinos, sendo raras as menções a cristais maiores, como em GOLDBERY \& LOUGHNAN (1977), que registraram a ocorrência de cristais quase milimétricos até cerca de $2 \mathrm{~mm}$, subeuédricos a euédricos, de pureza incomum. Sua associação freqüentemente resulta em glóbulos ou camadas botrioidais. A coloração varia bastante, tanto em função da composição dos fosfatos, como em função de sua possível estreita associação com outras fases, notadamente produtos ferruginosos.

Existe uma considerável dificuldade na determinação das características ópticas destes minerais, devido a sua ocorrência predominantemente em cristais de dimensões reduzidas e a suas associações a outras fases. Muitas vezes, não são nem mesmo diferenciados opticamente dos outros produtos co-existentes. Alguns autores chegaram a definir as seguintes características:

- birrefringência baixa e ausência de pleocroísmo, índices de refração variando de 1,605 a 1,620, para Sr-crandallita (LOUGHLIN \& SCHALLER 1917), 
TABELA 1 - Parâmetros de cela unitária de minerais da série da crandallita e compostos similares de origem sintética, apresentados por vários autores.

\begin{tabular}{|c|c|c|c|c|}
\hline \multirow{3}{*}{$\begin{array}{l}\text { Material } \\
\text { Crandallita sintética }\end{array}$} & \multicolumn{2}{|l|}{ Referência } & $\mathrm{a}(\AA)$ & $c(\AA)$ \\
\hline & \multicolumn{2}{|l|}{ SLADE (1974) } & 6,989 & 16,159 \\
\hline & \multicolumn{2}{|c|}{ GILKES \& PALMER (1983) } & 7,007 & 16,216 \\
\hline \multirow[t]{3}{*}{ Crandallita natural } & \multicolumn{2}{|c|}{ FRANSOLET \& DELIENS (1997) } & 6,999 & 16,671 \\
\hline & \multicolumn{2}{|l|}{ BLOUNT (1974) } & 7,005 & 16,192 \\
\hline & \multicolumn{2}{|c|}{ RADOSLOVICH (1969 apud SCHWAB } & 7003 & \\
\hline & & $\begin{array}{l}7,003 \\
7,013\end{array}$ & $\begin{array}{l}16,166 \\
16,196\end{array}$ \\
\hline & \multicolumn{2}{|c|}{ MITCHELL \& KNOWLTON (1971) } & 7,000 & 16,194 \\
\hline & \multirow{2}{*}{\multicolumn{2}{|c|}{$\begin{array}{l}\text { PALMER (1979, } \\
\text { apud GILKES \& PALMER, 1983) }\end{array}$}} & & \\
\hline & & & 7,005 & 16,192 \\
\hline Goyazita sintética & \multicolumn{2}{|l|}{ SCHWAB et al. $1990 \mathrm{a}$} & 7,024 & 16,611 \\
\hline & \multicolumn{2}{|c|}{ GILKES \& PALMER (1983) } & 7,013 & 16,650 \\
\hline \multirow[t]{5}{*}{ Goyazita natural } & \multicolumn{2}{|c|}{ KATO \& RADOSLOVICH $(1968$, apud } & & \\
\hline & \multicolumn{2}{|c|}{ GILKES \& PALMER 1983) / KATO (1971) } & 7,021 & 16,505 \\
\hline & \multicolumn{2}{|c|}{ GUILLEMAN (1955) } & 6,981 & 16,487 \\
\hline & \multicolumn{2}{|c|}{ MCKIE (1962) (Goyazita com Ce) } & 6,982 & 16,540 \\
\hline & \multicolumn{2}{|c|}{ PALACHE et al. (1951) } & 6,97 & 16,51 \\
\hline Gorceixita sintética & \multicolumn{2}{|l|}{ SCHWAB et al. 1990a } & 7,059 & 17,275 \\
\hline \multirow[t]{8}{*}{ Gorceixita natural } & \multicolumn{2}{|c|}{ KATO \& RADOSLOVICH $(1968$, apud } & & \\
\hline & GILKES \& PALMER 19 & 3) / KATO (1971) & 7,017 & 16,696 \\
\hline & RADOSLOVICH \& SI & $\operatorname{ADE}(1980)(*)$ & 7,0363 & 17,2819 \\
\hline & $\begin{array}{l}\text { KRESTEN \& CHYSSI } \\
\text { SCHWAB et al.1990a) }\end{array}$ & ER (1978 apud & 7,017 & 16,744 \\
\hline & TAYLOR et al. (1984) & & 7,02 & 17,29 \\
\hline & MCKIE (1962) & & 6,999 & 16,540 \\
\hline & MCKIE (1962) (Mrim & Hill) & 7,022 & 17,030 \\
\hline & MCKIE (1962) (Serra & eão) & 7,028 & 17,030 \\
\hline Plumbogummita sintética & SCHWAB et al. 1990a & & 7,033 & 16,789 \\
\hline & BAKER (apud SCHW & (B et al. 1990a) & 7,01 & 16,74 \\
\hline Plumbogummita natural & SLANSKY (1977) & & 7,017 & 16,75 \\
\hline & FÖRTSCH (1968) & & 7,018 & 16,784 \\
\hline Florencita sintética & SCHWAB et al. (1990 & ) $\mathrm{La}$ & 6,994 & 16,331 \\
\hline & SCHWAB et al. (1990 & $\mathrm{Ce}$ & 6,965 & 16,227 \\
\hline & SCHWAB et al. (1990 & Pr & 6,970 & 16,185 \\
\hline & SCHWAB et al. (1990 & $\mathrm{Nd}$ & 6,972 & 16,116 \\
\hline & SCHWAB et al. (1990 & $\mathrm{Sm}$ & 6,947 & 15,982 \\
\hline & SCHWAB et al. (1990 & $\mathrm{Eu}$ & 6,948 & 15,934 \\
\hline & SCHWAB et al. (1990 & $\mathrm{Gd}$ & 6,938 & 15,898 \\
\hline$" \quad c o m \mathrm{Sr}$ & SCHWAB et al. (1990 & $\mathrm{Sr} 0,3 \mathrm{Dy} 0,7$ & 7,019 & 16,601 \\
\hline & SCHWAB et al. (1990 & $\mathrm{Sr} 0,5 \mathrm{Er} 0,5$ & 7,019 & 16,591 \\
\hline & SCHWAB et al. (1990 & $\mathrm{Sr} 0,75 \mathrm{Lu} 0,25$ & 7,015 & 16,594 \\
\hline Florencita natural & POULIOT \& HOFMA & N (1981) & 6,99 & 16,25 \\
\hline & MCKIE (1962) & & 6,949 & 16,233 \\
\hline & LEFEBVRE \& GASP & RRINI (1980) & 6,987 & 16,248 \\
\hline$" \quad \operatorname{com~Sr}$ & MCKIE (1962) & & 6,971 & 16,420 \\
\hline & & $\mathrm{b}(\AA)$ & $c(\AA)$ & $\beta^{\circ}$ \\
\hline (*) Gorceixita natural & RADOSLOVICH \& & & & \\
\hline $\begin{array}{l}\text { calculada como } \\
\text { monoclínica }\end{array}$ & SLADE (1980) & 12,1892 & 7,0364 & 125,30 \\
\hline (*) Gorceixita natural & RADOSLOVICH \& & & & \\
\hline $\begin{array}{l}\text { calculada como } \\
\text { ortorrômbica }\end{array}$ & SLADE (1980) & 7,0478 & 9,9465 & - \\
\hline
\end{tabular}


- birrefringência baixa para gorceixita (TAYLOR et al. 1984),

- caráter uniaxial positivo, para crandallita (LARSEN \& SHANNON, 1930), para goyazita (SILVA \& VILLARROEL 1986) e para florencita,

- caráter isotrópico, ou quase, relevo moderado, índice de refração 1,596 para crandallita com Sr (BLANCHARD 1972),

- índices de refração de 1,719 a 1,713 para florencita (POGREBNOY et al. 1977) e 1,62 para gorceixita (COETZE \& EDWARDS 1959) e

- índice de refração entre 1,620 e 1,625 para gorceixita de Diamantina, Brasil (ATENCIO \& CLARK 1996) em amostra anteriormente identificada como ferrazita, nome desacreditado na mesma publicação citada.

Segundo NRIAGU (1984), os valores de densidade encontrados pelos diversos autores variam de 2,78 a 2,92 para crandallita, 3,46 a 3,71 para florencita, 3,26 para goyazita e 4,01 para plumbogummita.

Outra característica importante dos minerais desta série é a alta insolubilidade (BAIN 1970 e YOUNG 1958). WILSON (1984) recomenda a utilização de ácido fluorídrico para sua purificação.

\section{CARACTERÍSTICAS TÉRMICAS}

As características térmicas dos minerais da série da crandallita podem refletir sua composição, embora de uma forma ainda discutível conforme mostra a tabela 2, que reúne os dados apresentados na bibliografia consultada. De acordo com BLANCHARD $(1971,1972)$, a calcinação da crandallita acima de $600^{\circ} \mathrm{C}$ forma hidroxiapatita e "fosfocristobalita", enquanto whitelockita e coríndon formam-se a $1150^{\circ} \mathrm{C}$. Segundo GILKES \& PALMER (1979), crandallita altera-se em fase amorfa a $500^{\circ} \mathrm{C}$; sua calcinação, em mistura com outros minerais, como apatita e millisita, forma whitlockita, "fosfocristobalita" e "fosfotridimita" a $750^{\circ} \mathrm{C}$. Acima de $850^{\circ} \mathrm{C}$, forma-se coríndon. As diferenças encontradas entre estes autores devem ser atribuídas a condições diferentes nos ensaios.

De acordo com GILKES \& PALMER (1983), com o aumento da participação do $\mathrm{Sr}$ no sítio $\mathrm{A}$, a temperatura de desidratação diminui de $475^{\circ} \mathrm{C}$ para a crandallita a $420^{\circ} \mathrm{C}$ para crandallita com $20 \%$ molar de Sr; a temperatura de desidratação volta a aumentar para $440^{\circ} \mathrm{C}$ para a goyazita.

\section{OCORRÊNCIA E ORIGEM}

A alteração intempérica da apatita, principal fosfato primário presente nestes complexos, é geralmente o primeiro passo para a formação de fosfatos supérgenos nos perfis; esta alteração causa inicialmente modificações internas à sua estrutura e termina por promover sua dissolução. A evolução supérgena dos fosfatos primários, com lixiviação ou fixação do fósforo, e a formação dos fosfatos dependem de parâmetros como $\mathrm{pH}$, atividade do $\mathrm{H}_{3} \mathrm{PO}_{4}$ e dos cátions envolvidos nas novas fases ( $\mathrm{Na}, \mathrm{Ca}, \mathrm{Sr}, \mathrm{Ba}, \mathrm{Al}, \mathrm{Fe}$, ETR, $\mathrm{Pb}$ e ainda outros), segundo VIEILLARD et al. (1979) e SCHWAB et al. (1989). Estes parâmetros são função das características petrográficas, mineralógicas, geoquímicas e texturais das rochas envolvidas; os aspectos morfológicos e climáticos da área em questão também têm sua influência na determinação das condições físicoquímicas dos meios e, conseqüentemente, do comportamento dinâmico do fósforo.

Num estágio inicial de alteração de rochas apatíticas ricas em carbonatos (carbonatitos, calcários e fosforitos), soluções levemente ácidas permitiriam a dissolução dos carbonatos, originando um meio ligeiramente alcalino. A apatita, pouco solúvel neste meio, seria mantida estável; nos níveis onde os carbonatos já tiverem sido totalmente solubilizados, um meio levemente ácido favoreceria a sua dissolução $(5,5$ a 6,5 , segundo LUCAS et al. 1980, FLICOTEAUX \& LUCAS 1984, ZANIN 1989 e LOTTERMOSER 1990, entre outros).

São liberados então os íons constituintes, que não são prontamente lixiviados, sofrendo etapas de reciclagem, formando novas estruturas fosfáticas que se sucedem no tempo e no espaço, refletindo não só a evolução das condições com o aprofundamento do perfil, mas também possíveis variações nos controles da alteração ao longo do tempo. Outros fosfatos originais podem também sofrer a alteração que leva à formação dos fosfatos aluminosos da série da crandallita, destacando-se a monazita.

Após a dissolução dos fosfatos primários o fósforo liberado, nas diferentes formas dissocia- 
TABELA 2 - Características térmicas de minerais da série da crandallita, segundo a literatura consultada.

\begin{tabular}{|c|c|c|c|}
\hline Mineral (referência) & $\begin{array}{c}\text { efeitos } \\
\text { endotérmicos }\end{array}$ & $\begin{array}{c}\text { efeitos } \\
\text { exotérmicos } \\
\text { (oC) }\end{array}$ & $\begin{array}{c}\text { perda de } \\
\text { massa } \\
(\mathrm{oC})\end{array}$ \\
\hline \multicolumn{4}{|l|}{$\begin{array}{l}\text { Minerais da série da crandallita com ETR (sintéticos, } \\
\text { SCHWAB et al. 1990a): }\end{array}$} \\
\hline $\begin{array}{l}\mathrm{La} \mathrm{Al} 3\left(\mathrm{PO}_{4}\right)_{2}(\mathrm{OH})_{6} \\
\mathrm{Ce} \mathrm{Al} 3\left(\mathrm{PO}_{4}\right)_{2}(\mathrm{OH})_{6} \\
\mathrm{Pr} \mathrm{Al} 3\left(\mathrm{PO}_{4}\right)_{2}(\mathrm{OH})_{6} \\
\mathrm{Nd} \mathrm{Al} 3\left(\mathrm{PO}_{4}\right)_{2}(\mathrm{OH})_{6} \\
\mathrm{Sm} \mathrm{Al} 3\left(\mathrm{PO}_{4}\right)_{2}(\mathrm{OH})_{6} \\
\mathrm{Eu} \mathrm{Al} 3\left(\mathrm{PO}_{4}\right)_{2}(\mathrm{OH})_{6} \\
\mathrm{Gd} \mathrm{Al} 3\left(\mathrm{PO}_{4}\right)_{2}(\mathrm{OH})_{6}\end{array}$ & $\begin{array}{l}640 \\
650 \\
640 \\
630 \\
620 \\
610 \\
600\end{array}$ & & \\
\hline Goyazita sintética (SCHWAB et al, 1990b) & $520-560-690$ & 860 & \\
\hline Gorceixita sintética (SCHWAB et al. 1990b) & 560 & 870 & \\
\hline $\begin{array}{l}\text { Crandallita sintética } \mathrm{Cr}_{80} \mathrm{Go}_{20} \\
\text { (GILKES \& PALMER 1983) } \\
\text { Goyazita sintética (GILKES \& }\end{array}$ & 420 & & \\
\hline PALMER 1983) & $440(320)$ & & $15 \%\left(320^{\circ} \mathrm{C}\right)$ \\
\hline $\begin{array}{l}\text { Gorceixita natural (KRESTEN \& } \\
\text { CHYSSLER } 1978 \text { apud SCHWAB }\end{array}$ & & & \\
\hline $\begin{array}{l}\text { et al. 1990a) } \\
\text { Gorceixita natural (NICOLAS \& }\end{array}$ & $569-602$ & & \\
\hline DE ROSEN 1963) & 580 & & \\
\hline Gorceixita natural (RAO 1966) & $(110)-520$ & & \\
\hline Gorceixita natural (ANKINOWITCH \& & & & \\
\hline $\begin{array}{l}\text { SILANTJEVA } 1959 \text { apud SCHWAB } \\
\text { et al. 1990a) }\end{array}$ & $495-550$ & & \\
\hline Gorceixita natural (POVONDRA \& & & & \\
\hline $\begin{array}{l}\text { SLANSKY } 1966 \text { apud SCHWAB } \\
\text { et al. 1990a) }\end{array}$ & $500-700$ & & \\
\hline Crandallita (HILL et al. 1950) & 400 & & \\
\hline Crandallita (GILKES \& PALMER 1979) & 475 & & \\
\hline Crandallita (DOAK et al. 1965) & 475 & & \\
\hline Crandallita (COWGILL et al. 1963) & 512 & & \\
\hline Crandallita (BLANCHARD 1972) & 530 & & \\
\hline Florencita natural (MCKIE 1962): & & & \\
\hline $\mathrm{Sr}_{442} \mathrm{Ce}_{{ }_{52}} \mathrm{Ca}_{\cdot 14} \mathrm{Al}_{2.83}(\mathrm{PO} 4)_{21.77}\left(\mathrm{SO}_{4}\right) \cdot 1 \mathrm{~F}_{\cdot 41}(\mathrm{OH})_{5.24}\left(\mathrm{H}_{2} \mathrm{O}\right)_{85}$ & 628 & & \\
\hline $\mathrm{Ba}_{5} \mathrm{Sr}_{5} \mathrm{Ce}_{\cdot 41} \mathrm{Ca}_{08} \mathrm{Al}_{2.78}\left(\mathrm{PO}_{4}\right)_{1.62}(\mathrm{SO} 4)_{\cdot 19} \mathrm{~F}_{\cdot 3}(\mathrm{OH})_{5,3}\left(\mathrm{H}_{2} \mathrm{O}\right)_{1.18}$ & $636-655$ & & \\
\hline Plumbogummita sintética (SCHWAB et al. 1990b) & $510-870$ & $650-670$ & \\
\hline Plumbogummita sintética (BAKER 1963 apud & & & \\
\hline SCHWAB et al. 1990b) & $(140)-500$ & & \\
\hline Plumbogummita natural (RAO 1966) & 520 & & \\
\hline Plumbogummita (SLANSKY 1977) & $(120)-510$ & & \\
\hline
\end{tabular}

das do ácido fosfórico (VIEILLARD $1978 \mathrm{e}$ VIEILLARD et al. 1979) pode recristalizar-se dando origem predominantemente a dois tipos de estruturas: apatíticas e não apatíticas (onde se destacam os fosfatos da série da crandallita). $\mathrm{O}$ fósforo seria assim em parte fixado com outros cátions ém fases secundárias, e em outra parte migraria para níveis inferiores do perfil, even- tualmente formando apatita secundária ao atingir horizontes levemente alcalinos. Há, na literatura, menções do reconhecimento da assinatura geoquímica da apatita original nos fosfatos neoformados, em relação a vários elementos como ETR, Pb, U, Y, Mn, Zr, Nb e Sc (COSTA \& SIQUEIRA 1988, entre outros).

É importante ressaltar que a permanência, 
no perfil de alteração, do fósforo liberado pela dissolução dos fosfatos primários, tem grande influência na dinâmica de diversos elementos, já que os minerais fosfáticos, em particular os minerais da família alunita-jarosita (BOTTINELLY 1976 e SCOTT 1987), são estruturas favoráveis à fixação de elementos variados como $\mathrm{U}, \mathrm{Th}, \mathrm{V}, \mathrm{Sr}, \mathrm{Ba}, \mathrm{ETR}$ e outros já citados, alguns dos quais seriam lixiviados não fossem as neoformações fosfáticas.

O comportamento do fósforo no intemperismo pode então ser considerado residual em escala de perfil, mas com sucessivas reciclagens e mudanças de nível determinadas pelas variações das condições físico-químicas dos meios, resultando na zonalidade vertical comumente observada nos mantos de alteração laterítica, em termos de minerais fosfáticos secundários presentes.

Considerando os estudos geoquímicos efetuados por vários autores (ALTSCHULER 1973, VIEILLARD et al. 1979, LUCAS et al. 1980, SLANSKY 1980 e FLICOTEAUX \& LUCAS 1984, DILL et al. 1991 entre outros) em perfis de alteração laterítica sobre materiais com fosfatos que são progressivamente desestabilizados e reprecipitados, com mudanças na sua composição química, respondendo às condições físico-químicas do meio em cada etapa, pode-se admitir uma sequência típica de zonalidade vertical no perfil, quanto à formação de fosfatos secundários, com progressivo decréscimo em álcalis e hidratação e acréscimo da razão $\mathrm{Al}+\mathrm{Fe} / \mathrm{P}$, normalmente na seguinte ordem, da base para o topo:

$\mathrm{Fosf}_{1} \mathrm{Ca} \rightarrow \mathrm{Fosf}_{2} \mathrm{Ca} \rightarrow \mathrm{Fosf}_{3} \mathrm{Ca}, \mathrm{Al} \rightarrow \mathrm{Fosf}_{4} \mathrm{Al}, \mathrm{Fe}$ $\rightarrow$ Fosf $_{5} \mathrm{Al}+\mathrm{Fosf}_{6} \mathrm{Fe}$, onde:

$\mathrm{Fosf}_{1} \mathrm{Ca}$ e Fosf $\mathrm{F}_{2} \mathrm{Ca}$ : apatita primária e secundária $\mathrm{Fosf}_{3} \mathrm{Ca}$,Al: crandallita

$\mathrm{Fosf}_{4} \mathrm{Al}, \mathrm{Fe}$ : strengita com $\mathrm{Al}$

Fosf $f_{5}$ Al: variscita, wavelita, augelita e senegalita $\mathrm{Fosf}_{6} \mathrm{Fe}$ : strengita

Condições específicas de intemperismo e as disponibilidades geoquímicas particulares podem omitir algumas fases intermediárias na seqüência acima referida, como é o caso dos depósitos da região de Altay-Sayan, estudada por ZANIN (1968), onde a wavelita é encontrada com freqüência diretamente sobre a fase rica em fluorcarbonato apatita. Caso semelhante ocorre no Maciço Carbonatítico de Ipanema (SP), onde wavelita forma-se diretamente a partir da altera- ção da apatita primária (FLORÊNCIO 1995 e FLORÊNCIO \& TOLEDO 1997).

Nas alterações sobre materiais sedimentares portadores de argilominerais homogeneamente distribuídos (FLICOTEAUX 1982), fornecedores de $\mathrm{Al}$, em geral não se forma apatita secundária, o que somente ocorre quando os sedimentos não contêm $\mathrm{Al}$ ou o contêm em repartição heterogênea. Nestes locais, grande variedade de fosfatos aluminosos podem se formar, além dos pertencentes à série da crandallita. A presença dos íons $\mathrm{PO}_{4}{ }^{3-}$ no perfil inibe a cristalização da gibbsita, já que o alumínio tende a participar da estrutura dos fosfatos secundários. Fosfatos de ferro são mais raros na natureza que os fosfatos de alumínio, e isto seria explicado pelo fato de que as reações dos íons fosfatos com hidróxidos de ferro são muito mais lentas e ocorrem somente em ausência de material argiloso ou em condições muito redutoras; assim, o ferro permanece predominantemente como oxihidróxidos livres, ao contrário do alumínio, que forma gibbsita quando está em excesso em relação ao fósforo. Ainda em materiais sedimentares, na Bacia de Bauru, COUTINHO et al. (1999) estudaram uma ocorrência singular de fosfato aluminoso (gorceixita), como produto da substituição do fosfato de cálcio de ossos fósseis.

Os perfis de alteração intempérica sobre rochas de maciços carbonatíticos com fosfatos são geralmente ricos em fosfatos aluminosos e apresentam particular interesse em função de sua importância como material de cobertura das grandes jazidas fosfáticas do Brasil. Por sua heterogeneidade litológica e pela própria evolução do intemperismo, seguindo os controles determinantes específicos de cada local e de cada período, os mantos de alteração laterítica gerados sobre estes complexos apresentam-se muito heterogêneos. Exemplificando, os carbonatitos podem apresentar-se bem individualizados (exemplos brasileiros: Juquiá e Jacupiranga) ou não; os filossilicatos podem ser muito abundantes, determinando uma alteração em geral mais lenta (Ipanema) ou não; os fosfatos primários podem estar associados às fácies carbonatíticas (Jacupiranga e Juquiá) ou às fácies silicáticas (Ipanema, Catalão, Araxá, Anitápolis e Tapira), o que muda o meio geoquímico onde ocorre a alteração da apatita. Todos estes fatores, entre outros, desempenham seu papel na determinação da evolução supérgena dos fosfatos. 
A presença de fosfatos aluminosos desta série pode ser utilizada como indicador paleoclimático, como foi feito por CORREIA-NEVES et al. (1987), estudando a alteração de pegmatitos com ambligonita do nordeste de Minas Gerais; neste estudo, a presença de material crandallítico foi interpretada pelos autores como indicativa de uma fase de intemperismo laterítico, distinta do intemperismo atual.

\section{SÍNTESE DE COMPOSTOS DE ESTRUTURA CRANDALLÍTICA}

Os estudos de síntese geralmente visam à determinação das características dos termos extremos e intermediários da série, de composição controlada e homogênea, e servem de referência às discussões sobre os efeitos das várias substituições nas características das fases naturais, principalmente propriedades térmicas e parâmetros de cela unitária, como já foi visto.

SCHWAB et al. (1990a), em estudo de síntese dos termos puros goyazita, plumbogummita e gorceixita, em condições hidrotermais $\left(200^{\circ} \mathrm{C}\right.$ e 15 bar), concluíram ser impossível sintetizar o termo puramente cálcico, que se formaria apenas na presença de íons estranhos; no sistema cálcico puro, segundo aqueles autores, formamse preferencialmente hidroxiapatita, augelita e böhmita, indicando que a crandallita pura não teria estabilidade ou teria um domínio de estabilidade muito restrito, precisando de uma certa quantidade de íons maiores para estabilizar a estrutura. Em seu estudo, os autores determinaram as características mineralógicas e físicoquímicas das fases sintetizadas, incluindo os parâmetros de cela unitária e as temperaturas de desidratação. Concluíram que crandallita com cátions bivalentes apresenta desidratação em duas etapas; ao contrário, crandallita com cátions trivalentes teria tendência a apresentar desidratação em uma única etapa.

SCHWAB et al. (1990b) sintetizaram termos extremos da série, com apenas ETR na posição A (florencita), também em condições hidrotermais, a $200^{\circ} \mathrm{C}$ e 15 bar. Concluíram que florencita de ETR leves como $\mathrm{La}, \mathrm{Ce}$ e Pr é mais facilmente formada que a de ETR pesados, sendo que para ETR além do Gd, não ocorre a síntese. Destacaram o fato de que este comportamento é refletido na estabilidade térmica, com a temperatura de desidratação caindo continuamente desde florencita de Ce, La e Pr (cerca de $\left.650^{\circ} \mathrm{C}\right)$ até as fases com $\mathrm{Nd}$ a $\mathrm{Gd}\left(640^{\circ} \mathrm{C}\right.$ a $600^{\circ} \mathrm{C}$ ). Lantanídeos mais pesados que Gd somente podem ser admitidos na estrutura até uma certa concentração, desde que haja a participação de outros íons maiores estabilizadores como Sr ou Ce. A contração lantanídea deve ser responsável por este comportamento, com os ETR mais pesados e menores não se adaptando bem à posição $\mathrm{A}$. Destacam ainda que, em função deste comportamento, três grupos de crandallita de ETR podem ser distintos: um grupo com extremo enriquecimento em $\mathrm{La}, \mathrm{Ce}$ e $\mathrm{Pr}$, outro moderamente enriquecido em $\mathrm{Nd}$ a $\mathrm{Gd}$, e outro grupo com $\mathrm{Tb}$ a $\mathrm{Lu}$ apenas admitidos na estrutura.

Este estudo serve para demonstrar a diferença de habilidade dos diferentes cátions em participar das neoformações crandallíticas, sendo, no entanto, difícil correlacionar estas conclusões com os sistemas naturais. Estas considerações são particularmente interessantes no estudo dos fosfatos aluminosos dos mantos lateríticos sobre carbonatitos brasileiros, onde, apesar de haver grande disponibilidade de $\mathrm{Ca}$, ETR e Sr, além de $\mathrm{Ba}$, é este último que dá conta da maior parte da ocupação do sítio A, formando predominantemente gorceixita em várias partes dos perfís em Juquiá, Catalão e Tapira (TOLEDO et al. 1997, TOLEDO 1999 e FERRARI 2000).

\section{IMPORTÂNCIA DOS FOSFATOS DA SÉRIE DA CRANDALLITA}

Materiais originados pelo intemperismo sobre acumulações fosfáticas foram utilizados em experimentos envolvendo sua aplicação na indústria de fertilizantes. Embora os produtos de calcinação de aluminofosfatos sejam fonte menos efetiva de $\mathrm{P}$ para as plantas do que quantidades equivalentes de superfosfato, o esgotamento de reservas de minério apatítico ou o aumento de custos de sua lavra pode justificar o interesse nesta linha de investigação (GILKES \& PALMER 1979). A estas razões pode ser adicionada a preocupação com a liberação excessiva de $\mathrm{P}$ no ambiente a partir dos fertilizantes fosfatados tradicionais.

Segundo DOAK et al. (1965), nem todo fosfato aluminoso mostra aumento na liberação 
de P para as plantas com a calcinação. HILL et al. (1950), por exemplo, estudando material fosfático de Homeland, Florida, constituído por $41 \%$ de millisita e $23 \%$ de crandallita, mostraram que sua solubilidade em solução de citrato de amônio aumenta após calcinação, mas esta característica é revertida quando o material é umedecido. Ao contrário, material do Senegal (DOAK et al. 1965), quando calcinado e aplicado em testes agronômicos, promove razoável crescimento de plantas em solos fortemente calcários, sendo, entretanto, de ação pobre em solos ácidos. Estes mesmos autores (DOAK et al. 1965) estudaram os materiais fosfáticos derivados de guano de Christmas Island (Oceania); a laterização deste material resultou na concentração de oxihidróxidos de ferro e de alumínio e na formação de uma mistura de crandallita e millisita em proporções variáveis; a calcinação deste material a cerca de $450^{\circ} \mathrm{C}$ degradou sua estrutura cristalina, gerando um produto de maior solubilidade em termos de liberação de fosfato; sua mistura com superfosfato pode ainda trazer melhores resultados agronômicos.

GILKES \& PALMER (1979) citam os estudos de BUCHAN et al. (1970), ROBERT \& WHITE (1974) e BOXMA (1977), sobre os mesmos materiais, que apresentaram conclusões diversas quanto à avaliação da disponibilização do P para as plantas após o tratamento por calcinação. Em seu próprio estudo sobre o material de Christmas Island, aqueles autores (GILKES \& PALMER, 1979) concluíram que as propriedades mineralógicas e químicas dos produtos de calcinação poderiam explicar as variação nas quantidades de $\mathrm{P}$ solúvel em citrato para diferentes temperaturas de calcinação. Apontaram ainda a recristalização de crandallita com o umedecimento do produto calcinado como responsável pela reversão de suas propriedades de solubilidade.

Assim, além da potencialidade de uso na fabricação de fertilizantes, tornando possível o aproveitamento de concentrações fosfáticas formando os chamados "minérios marginais", os minerais da série da crandallita, por sua estabilidade em superfície e por suas características cristaloquímicas, podem, a exemplo da monazita e da apatita, ser pesquisados como fase imobilizadora de elementos indesejáveis no ambiente. Por outro lado, sua ocorrência natural tem uma grande influência na dinâmica de diversos elementos, modificando, ao menos temporariamente, o comportamento geoquímico de alguns dos elementos da posição $\mathrm{A}$, como $\mathrm{Ca}, \mathrm{Sr}$ e $\mathrm{Ba}$, notadamente, que são fixados nas estruturas crandallíticas, embora sejam, em princípio, considerados bastante móveis em superfície.

\section{REFERÊNCIAS BIBLIOGRÁFICAS}

ALCOVER NETO, A. \& TOLEDO, M.C.M. 1993. Evolução supérgena do carbonatito de Juquiá (SP). São Paulo: Revista do Instituto Geológico, 14(1): 31-43.

ALTSCHULER, Z.S. 1973. The weathering of phosphate deposits - Geochemical and environmental aspects. In: E.J. Griffith, A. Beeton, J.M. Spencer \& D.T. Mitchell (ed.) Environmental Phosphorus Handbook. N. York, John Wiley \& Sons, p. 33-95.

ATENCIO, D. \& CLARK, A.M. 1996. Ferrazite is identical to gorceixite. Mineralogical Magazine, 60: 841-842.

BAIN, D.C. 1970. Plumbogummite-Group Minerals from Mull and Morvern. Mineralogical Magazine, 37:934-938.

BLACKBURN, W.H. \& DENNEN, W.H. 1997. Encyclopedia of Mineral Names. Special
Publication 1 of The Canadian Mineralogist, Mineralogical Association of Canada, 360p.

BLANCHARD, F.N. 1971. Thermal analysis of crandallite. Quart. Journal of the Florida Academy Of Sciences, 34(1): 1-9.

1972. Physical and Chemical data for crandallite from Alachua County, Florida. American Mineralogist, 57: 473 - 484.

BLOUNT, A.M. 1974. The crystal structure of crandallite. American Mineralogist, 59: 41-47.

BOTINELLY, T. 1976. A review of the minerals of the alunite-jarosite, beudantite, and plumbogummite groups. Journal of Research USA Geological Survey, 4(2): 213-216. 
COETZE, G.L. \& EDWARDS, C.B. 1959. The Mrima Hill carbonatite, Coast Province, Kenya. Trans. Proc. Geol. Soc. South Africa, 62:373-395.

CORRÊA, S.L.A. 1996. Evolução gequímica das crostas lateríticas e dos sedimentos sobrepostos na estrutura de Seis Lagos (Amazonas). Centro de Geociências, Universidade Federal do Pará, Belém, Tese Doutorado, 160p.

CORREIA-NEVES, J.M.; MARCIANO, V.R.P.R.O.; LENA, J.C.; PEDROSASOARES, A.C. 1987. Fosfatos do tipo crandallita (plumbogummita, goyazita, gorceixita) resultantes do intemperismo de ambligonita de pegmatitos de Coronel Murta (nordeste de Minas Gerais) e seu significado paleoclimático. Revista Brasileira de Geociências, 17(1): 42-52.

CASSEDANNE, J.P. \& NEN, L.B.S. 1991. Strontian crandallite from the Alto Benedito pegmatite, Paraíba, Brazil. Mineralogical Record, 22: 183-185.

COSTA, M.L. 1984. A dinâmica de formação de lateritas: o exemplo do NE do Pará e NW do Maranhão. In: SBG, CONGRESSO BRASILEIRO DE GEOLOGIA, 33, Rio de Janeiro, Anais, p. 4823-4837.

\& SIQUEIRA, N.V.M. 1988. Correlações geoquímicas entre apatita $\mathrm{e}$ crandallita. Revista da Escola de Minas, UFOP. II Simpósio Brasileiro de Geoquímica. Ano LII, 41(1-4): 231-239.

COUTINHO, J.M.V; ATENCIO, D.; COIMBRA, A.M.; FERNANDES, L.A. 1999. Gorceixite, a singular product of replacement in fossil bones from the Bauru Basin, Brazil. Canadian Mineralogist, 37: 945-950.

COWGILL, U.M., HUTCHINSON, G.E. ; JOENSU, O. 1963. An apparently triclinic dimorph of crandallite from a tropical swamp sediment in El Petén, Guatemala. American Mineralogist, 48:1144-1153.

DEVISMES, P.; GUIGUES, J.; LAURENT, Y.; PARFENOFF, A. 1968. Première découverte de florencite en France. Bulletin de la Societé Française de Minéralogie et Cristallographie, 91: 500-502.

DILL, H.G.; BUSCH, K.; BLUM, N. 1991. Chemistry and origin of vein-like phosphate mineralization, Nuba Mountains (Sudan). Ore Geology Reviews, 6:9-24.
DOAK, B.W.; GALLAHER, P.J.; EVANS, L.; MULLER, F.B. 1965. Low temperature calcination of C-grade phosphate from Christmas Island. New Zealand Journal of Agricultural Research, 8:15-29.

FARRUGIA, L. J. 1997. Ortep-3 for Windows A version of ortep-iii with a graphical user interface (gui). Journal of Applied Crystallography, 30, 565.

FERRARI, V. C. 2000. Fosfatos primários e secundários nos perfis de intemperismo sobre os maciços alcalino-carbonatíticos de Juquiá (SP), Anitápolis (SC) e Tapira (MG). Instituto de Geociências, Universidade de São Paulo, São Paulo, Tese de Doutorado, 241p.

FLICOTEAUX, R. 1982. Genèse des phosphates alumineux du Sénégal Occidental. Etapes et guides d'altération. Thèse Doctorat d'Etat, Université d'Aix-Marseille III, Sciences Géologiques, 67, 227p.

\& LUCAS, J. 1984. Weathering of phosphate minerals. In: J.B. Nriagu \& P.B. Moore (ed.), Phosphate minerals. Springer-Verlag, Berlin-Heidelberg-New York-Tokyo, p.292-317.

FLORÊNCIO, R.V. de S. 1995. Estudo da alteração intempérica das rochas ricas em apatita da Mina Gonzaga de Campos, associadas ao Maciço alcalino-carbonatítico de Ipanema, SP. Instituto de Geociências, Universidade de São Paulo, São Paulo, Dissertação de Mestrado, 90 p.

\& TOLEDO, M.C.M. de 1997. Estudo da alteração intempérica sobre rochas ricas em apatita no Maciço alcalino-carbonatítico de Ipanema, SP. Geochimica Brasiliensis, 11(3): 261-284.

FÖRTSCH, E.B. 1968. Plumbogummite from Roughten Gill, Cumberland. Mineralogical Magazine, 36:530-538.

FRANSOLET, A.M. \& DELIENS, M. 1997. Crandallite et wardite dans les filons de quartz du Massif de Stavelot, Belgique. Bulletin de 1'Institut Royal des Sciences Naturelles de Belgique, Sciences de la Terre, 67: 189-194.

GILKES, R.J. \& PALMER, B. 1979. Calcined Christmas Island C-grade rock phosphate fertilizers: Mineralogical Properties, reversion and assessment by chemical extraction. Australian Journal of Soil Research, 17: 467-481. 
GILKES, R.J. \& PALMER, B. 1983. Synthesis, properties and dehydroxylation of members of the crandallite-goyazite series. Mineralogical Magazine, 47: 221-227.

GOLDBERY \& LOUGHNAN, F.C. 1977. Dawsonite, alumohydrocalcite, nordstrandite and gorceixite in Permian marine strata of the Sydney Basin, Australia. Sedimentology, 24:565-579.

GOREAUD, M. \& RAVEAU, B. 1980. Alunite and crandallite: a structure derived from that of pyrochlore. American Mineralogist, 65: 953-956.

GRIEFFO, W.; HERRMANN, K; MÜLLER, G.; STRAUSS, K.W. 1984. Sr-Gorceixite, a weathering product in rich iron ores from the Córrego do Feijão Mine, Minas Gerais, Brazil. Contributions in Mineralogy and Petrology, 87:418-419.

GUILLEMIN, C. 1955. Sur une varieté d'Hidoloite du Cap Garonne (Var). Bulletin de la Societé Française de Minéralogie et Cristallographie, 78:27-32

HENDRICKS, S.B. 1937. The crystal structure of Alunite and the Jarosites. American Mineralogist, 22:773-784.

HILL, W.L.; ARMIGER, W.H.; GOOCH, S.D. 1950. Some properties of pseudowavellite from Florida. Transactions of American Institute of Mining (Metall.) Engineering, 187: 699-702.

HOARE, J. 1980. In: The role of Phosphorous in Agriculture (Khasawneh, F.E., Sample, E.C. \& Kamprath, E.J., eds.) Madison, Wisconsin, American Society of Agronomy, 910p.

JAMBOR, J. L. 1999. Nomenclature of the alunite supergroup. Canadian Mineralogist, 37:1323-1341.

KATO, T. 1971. The crystal structures of goyazite and woodhouseite. Neues Jahrbuch Mineralogie Monatshefte, H6: 241-246.

1987. Further refinement of the goyazite structure. Short Communication, Mineral. J. 13(6): 390-396.

1990. The crystal structure of florencite. Neues Jahrbuch Mineralogie Monatshefte, H5: 227-231.

LARSEN, E.S. \& SHANNON, E.V. 1930. Two phosphates from Dehrn: dehernite and crandallite. American Mineralogist, 15: 303-306.
LEFEBVRE, J.J. \& GASPARRINI, C. 1980. Florencite, an occurrence in the Zairian Copperbelt. Canadian Mineralogist, 18: 301-311.

LEHR, J.R. 1967. Variations in composition of phosphate ores and related reactivity. Proc. 17 th Anual Meeting of the Fertilizer Industry, Washington, nov. 1967, p.61-67.

LEMOS, V.P. 1994. Evolução mineralógica e geoquímica de lateritos sobre rochas do complexo de Maicuru - Pará. Centro de Geociências, Universidade Federal do Pará, Belém, Tese de Doutorado, 180p.

LOUGHLIN, G.F. \& SCHALLER, W.T. 1917. Crandallite, a new mineral. American Journal of Science, [4], 43 (1): 69-74.

LOTTERMOSER, B.G. 1990. Rare-Earth element mineralization within the Mt. Weld carbonatite laterite, Western, Australia. Lithos, 24:151-67.

LUCAS, J.; FLICOTEAUX, R; NATHAN, Y.; PRÉVÔT, L.; SHAHAT, Y. 1980. Different aspects of phosphorite weathering. SEPM Special Publication № 29, p. 41-51.

MCKIE, D. 1962. Goyazite and florencite from two African carbonatites. Mineralogical Magazine, 33: 281-297.

MELFI, A.J.; SOUBIÈS, F.; TOLEDOGROKE, M.C. 1991. Comportamento do nióbio nas alterações das rochas do complexo alcalino-carbonatítico de Tapira, Minas Gerais. In: SBGq, CONGRESSO BRASILEIRO DE GEOQUÍMICA, 3, São Paulo, Anais, 1: 85-87.

MENCHETTI, S. \& SABELLI, C. 1976. Crystal chemistry of the alunite series: crystal structure refinement of alunite and synthetic jarosite. Neues Jahrbuch Mineralogie Monatshefte, H9: 406-417.

MICHEL, J.; COLE, K.H. ; MOORE, W.S. 1982. Uraniferous gorceixite in the South Carolina coastal plain (U.S.A.). Chemical Geology, 35: 227-245.

MITCHELL, R \& KNOWLTON, S. M. 1971. Crandallite from Gore, Frederick County, Virginia. Mineralogical Record, 2 (5):223-224.

NICOLAS, J. \& ROSEN, A. 1963. Phosphates hydrothermaux de basse température et kaolinisation: la gorceixite du massif des Colettes (Allier) et les minéraux associés 
(hinsdalite). Bulletin de la Societé Française de Minéralogie et Cristallographie, LXXXVI, 379-385.

NORRISH, K. 1968. Some phosphate minerals of soils. INTERNATIONAL CONGRESS OF SOIL SCIENCE, Trans. $9^{\text {th }}$ II: 713-723.

NRIAGU, J.O. \& MOORE, P.B. 1984. Phosphate Minerals. Springer-Verlag, Berlim, 442 p.

OLIVEIRA, N.P. \& COSTA, M.L. 1984. Os fosfatos aluminosos do Pará e do Maranhão: estágio atual de conhecimentos e estratégia para o aproveitamento econômico. Ciências da Terra, 10: 16-19.

PALACHE, C.; BERMAN, H.; FRONDEL, C. 1951. The System of Mineralogy (seventh ed.) 2. John Wiley and Sons, New York, $1.124 \mathrm{p}$.

PEREIRA, V.P. 1995. Alteração no Maciço Alcalino-carbonatítico de Catalão I (GO, Brasil). Evolução mineralógica. Porto Alegre, Curso de Pós-Graduação em Geociências, Universidade Federal do Rio Grande do Sul, Porto Alegre, Tese de Doutorado, $279 \mathrm{p}$.

POGREBNOI, V.T.; TIMOCHENKO, I.L.; KASPOUTINE, I.L. 1977. Crandallite à strontium et à terres rares des croutes d'altération des carbonatites. Minéralogie des dépots sédimentaires, 4: 74-79.

POULIOT, G. \& HOFMAN, H.J. 1981. Florencite: a first ocurrence in Canada. Canadian Mineralogist, 19: 535-540.

RADOSLOVICH, E.W. 1982. Refinement of gorceixite structure in $\mathrm{Cm}$. Neues Jahrbuch Mineralogie Monatshefte, 1982: 446-464.

\& SLADE, P.G. 1980. Pseudotrigonal symmetry and the structure of gorceixite. Neues Jahrbuch Mineralogie Monatshefte, H4: 157-170.

RAO, A.B. 1966. Note on DTA study of some rare Brazilian phosphate minerals. Mineralogical Magazine, 35:427-428.

RASMUSSEN, B. 1996. Early-diagenetic REE-phosphates minerals (florencite, gorceixite, crandallite, and xenotime) in marine sandstones. A major sink for oceanic phosphorous. American Journal of Science, 296, 16:601-632.

SCHWAB, R.G., HEROLD, H., COSTA, M.L. ; OLIVEIRA, N.P. 1989. The formation of aluminous phosphates through lateritic weathering of rocks. In: Balasubramanian, K.S. \& Evangelov, V.P. (ed.) Weathering: its products and deposits. Theophrastus, Athens. v. 2: 369-386.

SCHWAB, R.G.; HEROLD, H.; GÖTZ, C. ; OLIVEIRA, N.P. 1990a. Compounds of the crandallite type: Synthesis and properties of pure goyazite, gorceixite and plumbogummite. Neues Jahrbuch Mineralogie Monatshefte, H3:113-126.

SCHWAB, R.G.; HEROLD, H.; GÖTZ, C. \& OLIVEIRA, N.P. 1990b. Compounds of the crandallite type: Synthesis and properties of pure Rare Earth Element-phosphates. Neues Jahrbuch Mineralogie Monatshefte, H6: 241-254.

SCOTT, K.M. 1987. Solid solution in, and classification of, gossan-derived members of the alunite-jarosite family, northwest Queensland, Australia. American Mineralogist, 72: 178-187.

SILVA, R.R. \& VILLARROEL, H.S. 1986. Etude cristallographique de herderite et de goyazite de Frei Martinho, état de Paraíba, Brasil. Anais da Academia Brasileira de Ciências, 58(1):121-124.

SLADE, P. 1974. The synthesis of crandallite $\mathrm{CaAl}_{3} \mathrm{H}\left[(\mathrm{OH})_{6} /\left(\mathrm{PO}_{4}\right)_{2}\right]$. Neues Jahrbuch Mineralogie Monatshefte, 1974: 22-27.

SLANSKY, E. 1977. Plumbogummite from Ivanhoe Mine, Northern territory, Australia. Neues Jahrbuch Mineralogie Monatshefte, 1977:45-53.

SLANSKY, M. 1980. Géologie des phosphates sédimentaires. Mémoire Bureau de Recherche Géologique et Minière, $\mathrm{n}^{\circ}$ 114, 92p.

SOUBIÈS, F.; MELFI, A.J. ; AUTEFAGE, F. 1991. Comportamento geoquímico dos elementos terras raras nos alteritos da jazida de fosfato e titânio de Tapira (Minas Gerais, Brasil) - A importância dos fosfatos. Revista Brasileira de Geociências, 21(1) 3-16.

SVISERO, D.P.; MASCARENHAS, Y.P.; SIMONE, C.A. ; FRANCESCONI, R. 1984. Mineralogia da ocorrência de florencita do município de Bicas (MG). In: SBG, CONGRESSO BRASILEIRO DE GEOLOGIA, 33, Rio de Janeiro, Anais, p.4434-4441. 
TAYLOR, M.; SMITH, R.W.; AHLER, B.A. 1984. Gorceixite in Topaz Greisen Assemblage, Silvermine Area, Missouri. American Mineralogist, 69:984-986.

TOLEDO, M.C.M. de. 1999. Mineralogia dos principais fosfatos do Maciço Alcalinocarbonatítico de Catalão I (GO) e sua evolução no perfil laterítico. Instituto de Geociências, Universidade de São Paulo, São Paulo, Tese de Livre-Docência, 198p.

TOLEDO, M.C.M. de; FERRARI, V.C.; SANTOS, C.N. dos ; ALCOVER NETO, A. 1997. Aspectos geoquímicos da gênese dos fosfatos secundários associados ao Maciço Alcalino-carbonatítico de Juquiá (SP). In: SBGq, CONGRESSO BRASILEIRO DE GEOQUÍMICA, Salvador, Anais, p.392-395.

VAN WAMBEKE, L. 1972. Eyletterdite, un nouveau phosphate de thorium appartenant à la série de la crandallite. Bulletin de la Societé Française de Minéralogie et Cristallographie, 95: 98-105.

VIEILLARD, P. 1978. Géochimie des Phosphates. Etude thermodynamique. Application à la genèse et à l'altération des apatites. Sciences Géologiques, 51:1-181.

;TARDY, Y. ; NAHON, D. 1979. Stability fields and aluminum phosphates: parageneses in lateritic weathering of argilaceous phosphatic sediments. American Mineralogist, 64:626-634.

VISSER, D.; FELIUS, R.O. ; MOREE, M. 1997. Augelite and cerian crandallite in dumortierite quartzites, Vaca Morta quarry, Vereda range, Macaúbas, Bahia, Brasil. Mineralogical Magazine, 61: 607-609.

YOUNG, E.J. 1958. An occurrence of gorceixite in Arkansas. American Mineralogist, 43(7-8): 762-765.

WANG, R.; BRADLEY, W.F. ; STEINFINK, H. 1965. The crystal structure of alunite. Acta Crystalographica, 18:249-252.

WILSON, J.A. 1984. Crandallite-group minerals in the Helikian Athabasca Group in Alberta, Canada. Canadian Journal of Earth Science, 22:637-641.

ZANIN, YU. N. 1968. Zones of lateritic weathering of secondary phosphorites of Altay-Sayan region. International Geological Review, 10 (10):1119-1127.

1989. Phosphate-bearing weathering crusts and their related deposits. Weathering: its products and deposits. vol. II. Products - Deposits - Geotechnics, p. 321-367, Theophrastus Publications, S.A.. Zographon, Athens, Greece.

Endereço da autora:

Maria Cristina Motta de TOLEDO - Instituto de Geociências (Departamento de Geologia Sedimentar e Ambiental) e NUPEGEL, Universidade de São Paulo, Rua do Lago, 562, CEP 05508-900 São Paulo - SP Brasil. E-mail: mcristol@usp.br 